\title{
Deployment and Selection of Monitoring Nodes for Detection of Selfish Attacks in MANET
}

\author{
R. Mangayarkarasi, R. Manikandan
}

\begin{abstract}
Since Mobile Ad hoc Network (MANET) has distributed network structure using wireless links, designing efficient security applications has become a critical need. Selfish nodes are nodes that refuse to forward the data from other nodes. The existence of selfish nodes will disturb the normal process of the network, and reduce the network performance. Intrusion Detection System (IDS) is a scheme for detecting any misbehaviors in the network operation by monitoring the traffic flow. Each monitoring node need to execute the IDS module. The common problems encountered by the monitoring nodes are energy depletion, link disconnection, mobility and coverage. Hence the selection of monitoring nodes plays an important role in IDS. This paper develops a technique for deployment and selection of monitoring nodes for detection of selfish attacks. In this technique, the whole network is virtually divided in smaller grid like zones. In each grid, the nodes with higher stability and better coverage are assigned a reward value. A cost metric is derived in terms of energy consumption and computational delay. Then the nodes with minimum cost and high reward are selected as monitoring nodes. By simulation results, it is shown that the proposed technique has reduced detection delay, energy consumption and detection overhead.
\end{abstract}

Keywords: MANET; DoS; Selfish Attack; IDS; misbehavior

\section{INTRODUCTION}

Mobile Ad hoc Network (MANET) is fundamentally a selforganizing network composed of plentiful nodules which are proficient of great flexibility and are associated to each other in a wireless method. In MANET, every mobile node is proficient of working as a router [1]. Since MANET has a distributed network structure using wireless links, several security threats are faced. As a result, designing efficient security applications has become a critical need in MANET [2][3]. Some of the control traffic attacks are Sybil attack, wormhole attack, rushing attack, etc [4]. Some of the major data traffic attacks are selective forwarding attack, blackhole attack, delaying and misrouting attack, etc [5]. One of the harmful attack is Denial of Service (DoS) attack. It exhausts the victim's network resources such as bandwidth, computing power, battery etc [6]. In Sybil attack, an attacker can create more than one identity on a single physical device in by launching a coordinated attack on the network [7]. Among these attacks, DoS, packet dropping attack and cheating attack are considered in this paper.

Revised Manuscript Received on March, 282020.

* Correspondence Author

R. Mangayarkarasi*, Assistant Professor, Department of Computer

Science, Government Arts College, $\quad$ Trichy. E-mail: Mangayarkarasiphd@gmail.com

Dr. R. Manikandan, Associate Professor, Department of Computer Science and Engineering, Sengipatti, Thanjavur. E-mail: rmkmanikandan@yahoo.co.uk

(C) The Authors. Published by Blue Eyes Intelligence Engineering and Sciences Publication (BEIESP). This is an open access article under the CC BY-NC-ND license (http://creativecommons.org/licenses/by-nc-nd/4.0/)
Intrusion detection is described as a process of identifying any actions or series of actions which can affect the The selfish nodes are nodes that refuse to forward the data from other nodes. The existence of selfish nodes will disturb the normal process of the network, and reduce the network performance. confidentiality, integrity or availability of a network resource. Intrusion Detection System (IDS) is a scheme for detecting any misbehaviour in the network operation by monitoring the traffic flow [8]. In MANET, IDS should be developed in order to detect any active or passive attack and then respond to it by using appropriate defense scheme [9]. Each monitoring node need to execute the IDS module. Hence the selection of monitoring nodes plays an important role in IDS. The common problems encountered by the monitoring nodes are energy depletion, link disconnection, mobility and coverage. Hence this paper develops a technique for deployment and selection of monitoring nodes for detection of selfish attacks. The paper is organized as follows. In section 2, few of the existing works are discussed. Section 3 presents the detailed description of proposed methodology. In section 4, simulation results and analysis are described. Section 5 concludes the paper.

\section{RELATED WORKS}

Mustafa et al [9] have presented a distributed and cooperative mechanism for detecting routing attacks in MANETs. In this mechanism, both the neighbor nodes as well as remote node are taken into consideration, and then its direct as well as indirect network factors are monitored to develop behavior characteristics. The anomalous event is detected based on the routing delay time duration as well as packet count. But, gathering information related to the delay involved in packet transmission through all the paths will create high overhead. Also, only the forwarded packets and received packets are taken into consideration for developing the behavior metrics and all the remaining layer metrics are not considered. Balan et al [10] have proposed a Fuzzy based IDS to detect the malicious behaviour of nodes as well as to determine the attack type. This proposed technique is appropriate for mainly two kinds of attack: black hole attack and the gray hole attack. In this paper, just packet drop is considered as the major factor for detection of black hole nodes. However, precise result will not be attained from it since it will give rise to many false positive cases. Subha et al. [11] have presented a game theory based IDS mechanism that includes a cluster leader election procedure and a hybrid IDS. The hybrid IDS consists of a threshold based lightweight unit and also an anomaly based heavyweight unit.

Published By:

Blue Eyes Intelligence Engineering

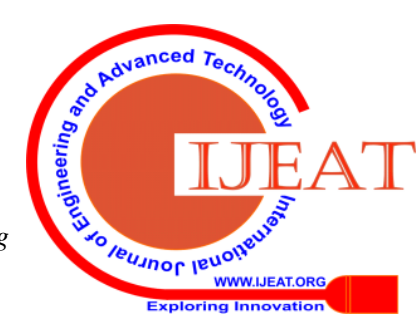


The lightweight unit is developed based on the Packet Forwarding Rate (PFR) factor. The normal network profile is developed based on the unsupervised association-rule mining technique and employed in the heavy weight module. In the Bayesian game model, the cluster leader is permitted to design its monitoring technique. Imani et al. [12] have presented a combined technique for misuse detection along with the anomaly detection to examine the network and determine the kind of attack. In this technique, the combination issue is resolved by employing the partially observed Markov decision process (POMDP). Whenever the known attack probability is greater, the misuse detection scheme is used. The anomaly detection scheme is used, whenever the unknown attack probability is greater and crosses the threshold value.

Vali et al. [13] have suggested a Cross-layer centered dispersed and supportive IDS by means of Dempster-Shafer indication philosophy. The network action is frequently observed by the indigenous discovery engine. It activates the IDS whenever any malicious behavior is seen. But, just the packet dropping attack is taken into consideration in this paper.

\section{PROPOSED SOLUTION}

\section{1 overview}

In this technique, the whole network is virtually divided in smaller grid like zones. The entire nodules in every lattice are inspected and the nodules with advanced steadiness and better coverage are assigned a reward value. A cost metric is derived in terms of energy consumption and computational delay. Then the nodes with minimum cost and high reward are selected as monitoring nodes.

\section{2 node stability}

For foreseeing the forthcoming state of the network, the flexibility is assessed which is well-defined as follows.

$\mathrm{S}_{\mathrm{i}}=\frac{1}{\Delta t}\left|\operatorname{Davg}_{i}(t)-\operatorname{Davg}_{i}(t+\Delta t)\right|$

$$
\operatorname{Davg}_{\mathrm{i}}=\frac{1}{n} \sum_{j=1}^{n} D_{(i, j)}(t)
$$

$$
\mathrm{D}_{(\mathrm{i}, \mathrm{j})}=\sqrt{\left(x_{i}-x_{j}\right)^{2}+\left(y_{i}-y_{j}\right)^{2}}
$$
the nodules

Where Si signifies the comparative rapidity of

Davgi(t) signifies the average expanse amongst the nodule $\mathrm{i}$ and its

adjacent at period $t$.

$\mathrm{n}$ is the number of adjacent nodule $\mathrm{i}$ $\mathrm{D}(\mathrm{i}, \mathrm{j})(\mathrm{t})$ is the expanse amongst nodule $\mathrm{i}$ and nodule j.

$\mathrm{x}$ and $\mathrm{y}$ signify the synchronizes of the nodule. Dependability of a wireless association is able to be dignified by means of LET which is a location centered layer metric. The movement factors of two adjacent nodules are vital for open space broadcast. Hence, in MANETs, global positioning system (GPS) is desirable by every

nodule. The period for which these two nodules are associated is evaluated by means of the movement factors of two nodules. Here it is presumed that nodules have equivalent broadcast radius y with particular locations ( $m 1$, $n 1)$ and $(m 2, n 2)$. The rapidity along the directions $d 1$ and $d 2$ are signified as $\mathrm{s} 1$ and $\mathrm{s} 2$. The succeeding equation offers the LET calculation.

$$
L E T=\frac{-(p q+r s)+\sqrt{\left(p^{2}+r^{2}\right) y^{2}-(p s-q r)^{2}}}{\left(p^{2}+r^{2}\right)}
$$

$$
\begin{aligned}
& \mathrm{p}=\mathrm{s} 1 \cos d 1-\mathrm{s} 2 \cos d 2 \\
& \mathrm{q}=\mathrm{m} 1-\mathrm{m} 2 \\
& \mathrm{r}=\mathrm{s} 1 \sin d 1-\mathrm{s} 2 \sin d 2 \\
& \mathrm{~s}=\mathrm{n} 1-\mathrm{n} 2
\end{aligned}
$$

\subsection{ENERGY CONSUMPTION}

Let $E_{i}$ be the preliminary energy of a nodule After the time period $t$, the energy spent by the nodule ( $\mathrm{E}$ (t)) is provided by means of succeeding equation [4]

$$
\mathrm{E}(\mathrm{t})=\mathrm{n}_{\mathrm{tx}} * \varepsilon+\mathrm{n}_{\mathrm{rx}} * \delta
$$

where $n_{\mathrm{tx}}$ and $\mathrm{n}_{\mathrm{rx}}$ are the number of data packages transferred and obtained by the nodule after time $\mathrm{t}$.

$$
\varepsilon \text { and } \delta \text { are coefficients in the series }(0,1)
$$

\subsection{Cost of a Node}

Let $\mathrm{dc}_{\mathrm{i}}$ be the computation delay of node $\mathrm{N}_{\mathrm{i}}$.

Then the cost of a node is computed in terms of energy consumption and computational delay as

$$
\mathrm{C}_{\mathrm{i}}=\mathrm{E}_{\mathrm{i}}+\mathrm{dc}_{\mathrm{i}}
$$

where $C_{i}$ and $E_{i}$ are the cost and energy consumption of node $\mathrm{Ni}$.

\subsection{Reward of a Node}

Primarily the entire nodules of the network are allotted a recompense value $\mathrm{RW}$, universally.

\subsection{Deployment of Monitoring Nodes}

The entire nodules in MANET are inspected and the nodules with advanced steadiness and remaining energy are nominated and are denoted as Monitoring nodules (MNs). These MNs will further be responsible for monitoring the network within its transmission range and determining the trust value of these surrounding nodes, in order to ensure the authenticity of these nodes. The process of selecting the MNs is described in algorithm 1.

\section{Algorithm - Deploying and Selecting MNs_}

Notations Definition

$\mathrm{G}_{1}, \mathrm{G}_{2}, \ldots \mathrm{G}_{\mathrm{k}}$

$\mathrm{MN}_{1}, \mathrm{MN}_{2}, \ldots . \mathrm{MN}_{\mathrm{k}}$

$\mathrm{D}_{\text {im }}$

grid $\mathrm{G}_{\mathrm{i}}$

Average $\left\{D_{i m}\right\}$

$\mathrm{Rw}_{\mathrm{i}}$
Virtual grids

Monitoring nodes

Node density of each

Average node density all grids Reward value of $\mathrm{MN}_{\mathrm{i}}$.

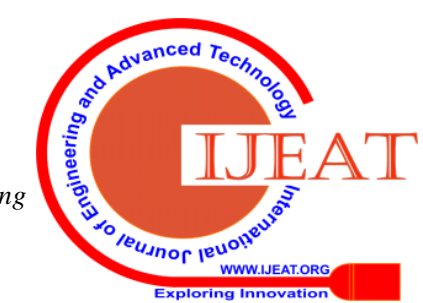


$\mathrm{C}_{\mathrm{i}}$

inc

reward and cost

$\mathrm{d}_{\mathrm{i}}$

$\operatorname{MinE}_{\mathrm{r}}$

value for residual energy

MinLET

LET

$\mathrm{Ne}\left(\mathrm{MN}_{\mathrm{j}}\right)$

$\mathrm{MN}_{\mathrm{j}}$

1. Split the network effectively into $\mathrm{k}$ smaller grids $\mathrm{G}_{1}, \mathrm{G}_{2}, \ldots . \mathrm{G}_{\mathrm{k}}$

2. For each $\mathrm{G}_{\mathrm{i}}, \mathrm{i}=1,2 \ldots \mathrm{k}$.

3. Choose any $\mathrm{MN}_{\mathrm{j}} \in \mathrm{G}_{\mathrm{i}}$

4. Estimate $D_{\text {im }}$ of $G_{i}$

5. $\quad$ Estimate $\mathrm{C}_{\mathrm{i}}$

6. If $\mathrm{D}_{\mathrm{im}}>$ Average $\left\{\mathrm{N}_{\mathrm{im}}\right\}$ and LET $\left(\mathrm{MN}_{\mathrm{j}}\right)>$ MinLET then

7. $\quad \mathrm{Rw}_{\mathrm{i}}=\mathrm{Rw}_{\mathrm{i}}+$ inc

8. If $\mathrm{C}_{\mathrm{i}}=\operatorname{Minimum}\left(\mathrm{C}_{\mathrm{i}}\right)$ and $\mathrm{Rw}_{\mathrm{i}}=$ $\operatorname{Maximum}\left(\mathrm{R}_{\mathrm{i}}\right)$, then

9. $\mathrm{MN}_{\mathrm{j}}$ is selected

10. Else

11.

12.

13.

14. Else

15. Find $\mathrm{G}_{\mathrm{k}}=\mathrm{G}_{\mathrm{i}} \cup \mathrm{G}_{\mathrm{i}+1}$

16.

17. End if

Choose $\mathrm{Ne}\left(\mathrm{MN}_{\mathrm{j}}\right)$

Repeat from step 4. End if

Cost of $\mathrm{MN}_{\mathrm{i}}$

delay of $\mathrm{MN}_{\mathrm{i}}$

Minimum threshold

Nearest neighbor of

\section{End For}

In this algorithm, the whole network is virtually divided in smaller grid like zones for convenience. Initially, one node $\mathrm{N}_{\mathrm{j}}$ is randomly selected in each zone. It estimates node density of its zone. The decision for a node to act as a MN will be on the basis of the high dense zone. If the node density of that zone is more than the average value, the selected node will be further checked for LET and residual energy. If both are higher than the minimum threshold values, then the node $\mathrm{N}_{\mathrm{j}}$ is selected as $\mathrm{MN}$. Otherwise, the nearest neighbor node of $\mathrm{Nj}$ is considered and checked for stability and energy conditions. This process is continued until a node from the same zone, satisfying the conditions is found. On the other hand, if the node density is less, the next zone will be merged to form a new larger zone, and the process is repeated.

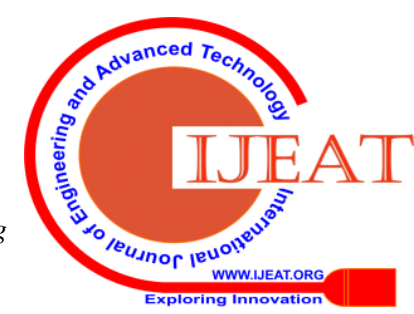




\subsection{Threat Model}

We take into consideration a MANET milieu in which both an outward challenger (outsiders) and inside (prevailing members) challenger, bout the multicast congestion. The deliberated bouts are DoS and package plummeting bouts.

\subsection{Results \& Analysis}

\section{Varying the nodes with $5 \%$ of attackers}

In order to analyze the effect of attackers over the network size, the number of nodes is varied as 50,75,100,125 and 150 with $5 \%$ of the nodes as attackers.

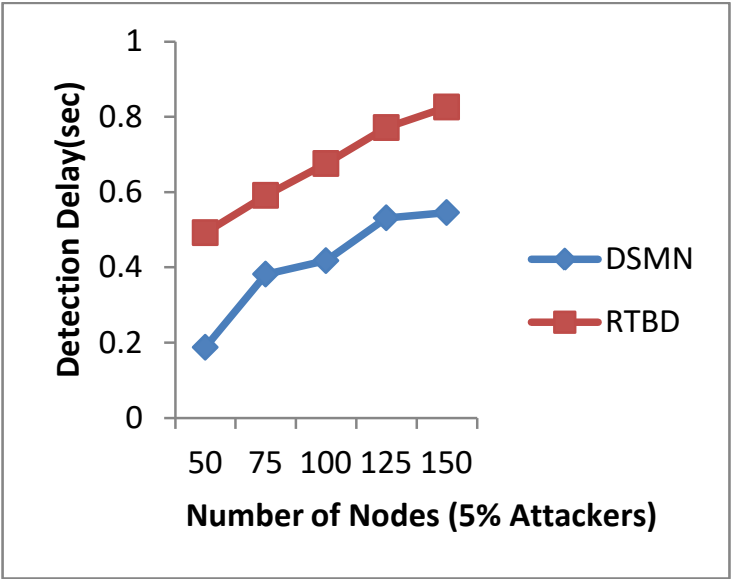

Figure 2 Detection Delay for $5 \%$ of attackers

The result of detection delay of DSMN and RTBD techniques is presented in Figure 2.As the nodes are increased, the delay of DSMN increases from 0.18 seconds to 0.54 seconds whereas the detection delay of RTBD increases from 0.49 to 0.82 seconds. Since the attacks are detected quickly by collaborative detection of monitoring nodes, the detection delay of DSMN is $40 \%$ lesser than RTBD.

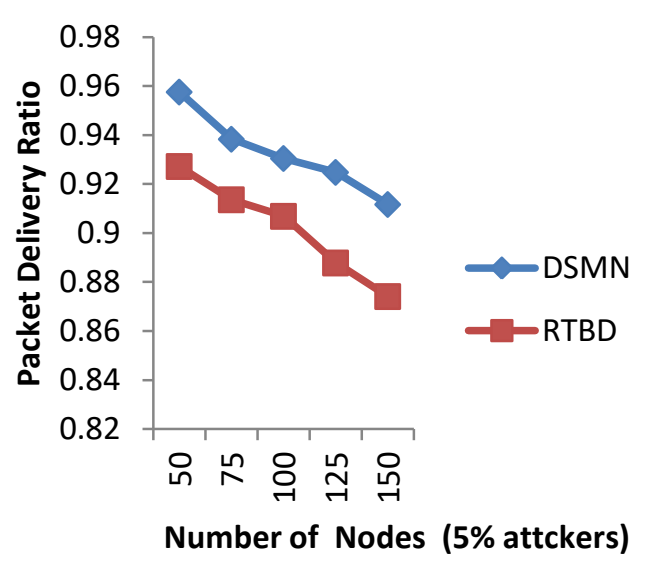

Figure 3 Packet delivery ratio for $5 \%$ of attackers

The result of packet delivery ratio of DSMN and RTBD techniques is presented in Figure 3. As the nodes are increased, the delivery ratio of RTBD decreases from 0.95 to 0.91 and the delivery ratio of RTBD decreases from 0.92 to 0.87 . As DSMN detects attacks on both network and MAC layers, the delivery ratio of DSMN is $3 \%$ higher than RTBD.

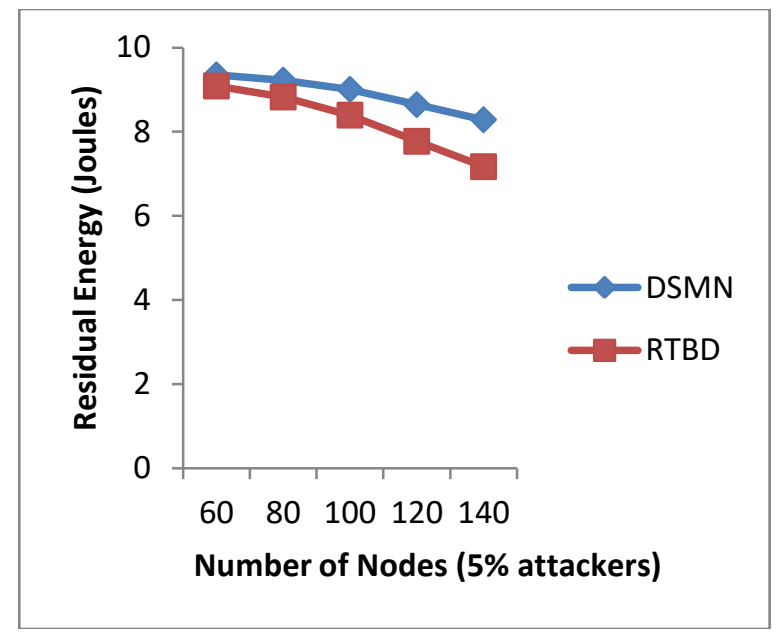

Figure 4 Residual Energy for 5\% of attackers

The residual energy of DSMN and RTBD techniques is shown in Figure.4. As the nodes are increased, the residual energy of DSMN decreases from 9.3 to 8.2 joules and residual energy of RTBD decreases from 9.0 to 7.1 joules. Since DSMN aims to detect attacks on energy depletion, it has 7.5\% higher residual energy than RTBD.

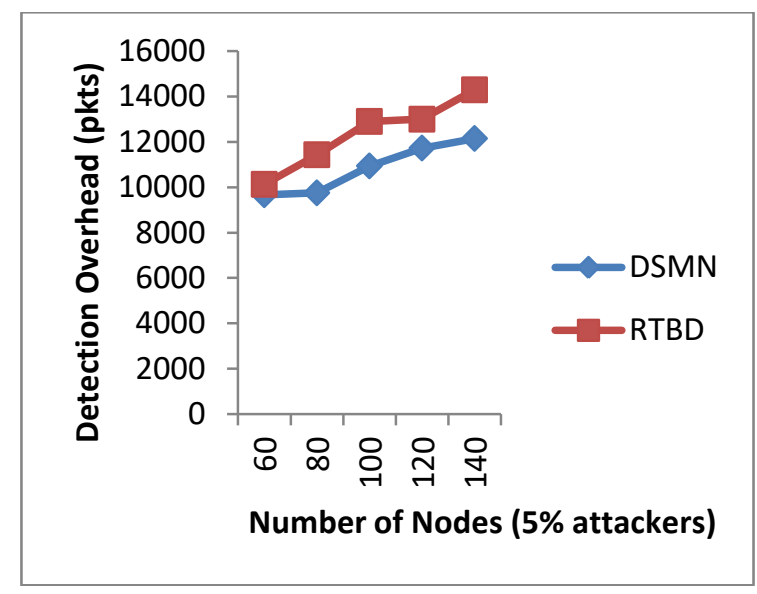

Figure 5 Detection Overhead for $5 \%$ of attackers

The detection overhead of DSMN and RTBD techniques is shows in Figure 5. As the nodes are increased, the overhead of DSMN increases from 9664 to 12144 packets and overhead of RTBD increases from 10125 to 14285 packets. Since the stable and energy efficient nodes are selected for monitoring, the frequency of changing the monitoring node is less. Hence the detection overhead of DSMN is $11 \%$ less when compared to RTBD.

\section{CONCLUSION}

This paper develops a technique for deployment and selection of monitoring nodes for detection of selfish attacks. In this technique, the whole network is virtually divided in smaller grid like zones. In each grid, the nodes with higher stability and better coverage are assigned a reward value. A cost metric is derived in terms of energy consumption and computational delay.

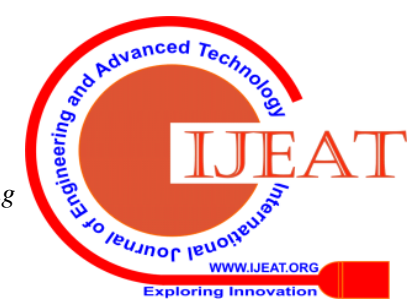


Then the nodes with minimum cost and high reward are selected as monitoring nodes. By simulation results it has been shown that the proposed DSMN technique has reduced detection delay, energy consumption and detection overhead.

\section{REFERENCES}

1. Usha G, M. Rajesh Babu, S. Saravana Kumar,(2016), "Dynamic anomaly detection using cross layer security in MANET", Computers and Electrical Engineering, Elsevier, 1-11.

2. Javidi M.M and Laya Aliahmadipour,(2015),"Game Theory Approaches in Taxonomy of Intrusion Detection for MANETs",Computer Engineering and Applications Vol. 4, No. 1.

3. Amiria E., Hassan Keshavarz, Hossein Heidari, Esmaeil Mohamadi, Hossein Moradzadeh,(2014), "Intrusion Detection Systems in MANET: A Review",Procedia - Social and Behavioral Sciences, 129, $453-459$.

4. Khan M.S and Noor M. Khan,(2016), "Low Complexity Signed Response Based Sybil Attack Detection Mechanism in Wireless Sensor Networks",Journal of Sensors,Hindawi Publishing Corporation, Article ID 9783072, 9 pages

5. Behzad S.S.J.,(2015), “A Survey over Black hole Attack Detection in Mobile Ad hoc Network", International Journal of Computer Science and Network Security,44, VOL.15 No.3.

6. .Sari A.,(2014),"Security Approaches in IEEE 802.11 MANET", Int. J. Communications, Network and System Sciences,7, 365-372

7. Abbas S., Madjid Merabti, David Llewellyn-Jones, and Kashif Kifayat, (2013),"Lightweight Sybil Attack Detection in MANETs", IEEE SYSTEMS JOURNAL, VOL. 7, NO. 2.

8. Abdalla A.M, Imane A. Saroit, Amira Kotb, Ali H. Afsari,(2011),"Misbehavior Nodes Detection and Isolation for MANETs OLSR Protocol",Procedia Computer Science 3,115-121

9. Mustafa H, Yan Xiong, Khalid Elaalim,(2014), "Distributed and Cooperative Anomaly Detection Scheme for Mobile Ad Hoc Networks", Journal of Computer and Communications, 2, 1-10

10. Balan V.E, Priyan M K, Gokulnath C, Prof.Usha Devi G,(2015), "Fuzzy Based Intrusion Detection Systems in MANET", Procedia Computer Science, 50, 109 - 114

11. Subba B., Santosh Biswas, Sushanta Karmakar,(2016),"Intrusion detection in Mobile Ad-hoc Networks: Bayesian game formulation",Engineering Science and Technology, an International Journal, Elsevier, 19,782-799

12. Imani M, Mohammad Ebrahim Rajabi, Mahdi Taheri, and Majid Naderi,(2015),"A Novel Approach to Combine Misuse Detection and Anomaly Detection Using POMDP in Mobile Ad-Hoc Networks",International Journal of Information and Electronics Engineering, Vol. 5, No. 4.

13. Vali Y.S And T.R.Rangaswamy,(2017),"An Efficient Cross-Layer Based Intrusion Detection System For Mobile Ad Hoc Networks", Journal of Theoretical and Applied Information Technology, Vol.95. No.1

14. Subramaniyan S., William Johnson and Karthikeyan Subramaniyan,(2014), "A distributed framework for detecting selfish nodes in MANET using Record- and Trust-Based Detection (RTBD) technique", EURASIP Journal on Wireless Communications and Networking, 2014:s205

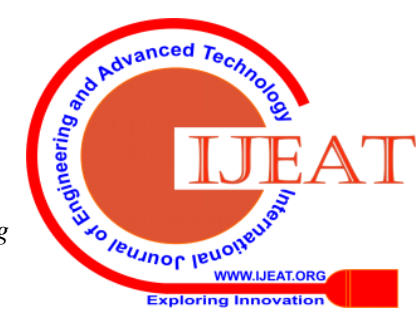

